\title{
ОПТИМИЗАЦИЯ СТРУКТУРЫ СЕТИ СТАНЦИЙ ЭКОЛОГИЧЕСКОГО МОНИТОРИНГА
}

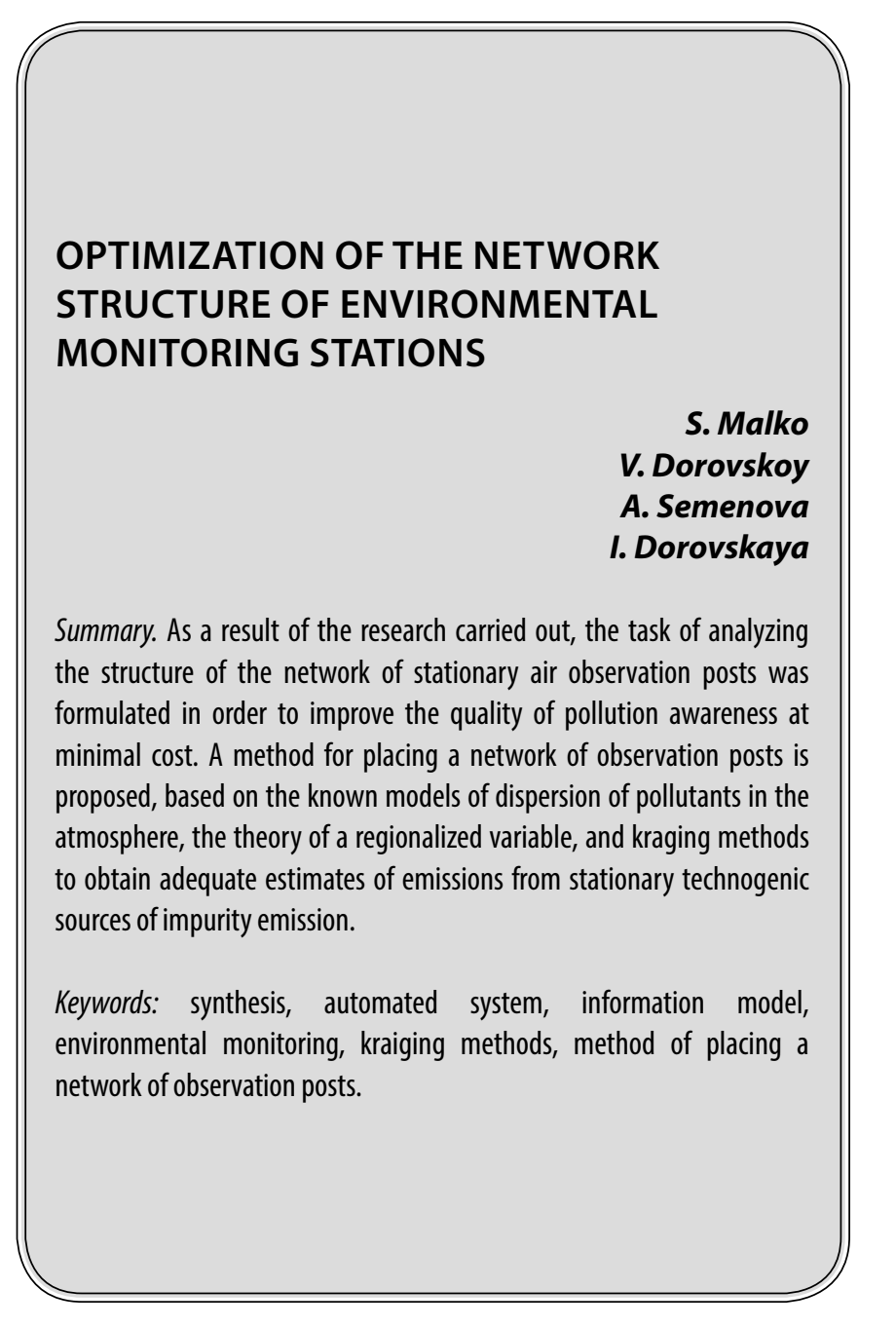

\section{Актуальность темы исслеАований}

$\mathbf{M}$ ониторинг стационарных источников загрязнения частично может вестись с использованием стационарных постов контроля состояния атмосферного воздуха [1, 2, 5-8]. В связи с высокой стоимостью внедрения и эксплуатации таких постов, а также стохастическим характером распространения примеси в атмосфере, эффективность работы группы стационарных постов региона существенно зависит от расположения их в пространстве [6]. С одной стороны, необходимо выбрать такое количество постов и их размещение, чтобы в течение времени нормирования наблюдений вероятность обнаружения концентраций выбросов, превышающих ПДК, была не менее установленного доверительного интервала (обычно выбирают 95\% веро-
Малько Сергей Владимирович

К.б.н., дочент, ФГБОУВО «Керченский государственный морской технологический университет», г. Керчь sergmalko@mail.ru

Доровской Владимир Алексеевич Д.т.н., профессор, ФГБОУ ВО «Керченский государственный морской технологический университет», г. Керчь

Семенова Анна Юрьевна

К.э.н., дочент, ФГБОУВО «Керченский государственный морской технологический университет», г. Керчь

Доровская Ирина Дмитриевна ФГБОУ ВО «Керченский государственный морской технологический университет», г. Керчь

Аннотация. В результате проведенных исследований сформулирована задача анализа структуры сети стационарных постов наблюдения атмосферного воздуха с целью повышения качества информированности 0 загрязнении при минимальных затратах. Предложен метод размещения сети постов наблюдения, основанный на известных моделях рассеяния примеси в атмосфере, теории о регионализованной переменной и методах крайгинга для получения адекватных оценок о выбросах стационарных техногенных источников эмиссии примеси.

Ключевые слова: синтез, автоматизированная система, информационная модель, экологический мониторинг, методы крайгинга, метод размещения сети постов наблюдения.

ятность обнаружения для совокупности стационарных постов), с другой стороны, стоимость эксплуатации всех постов в течение времени нормирования не должна превышать ожидаемый ущерб от детерминированных источников загрязнения, иначе использование таких постов экономически нецелесообразно. Поэтому целью работы является формулирование задачи анализа структуры сети стационарных постов наблюдения атмосферного воздуха для повышения качества информированности о загрязнении при минимальных затратах.

\section{Постановка задачи}

Пусть $m$ существующих источников выбросов размещены в различных точках $P_{l}, P_{2}, \ldots P_{m}$ пространства, а $n$ стационарных постов наблюдения - в точках $X_{1}, X_{2}, \ldots$ 
$X_{n}$. Обозначим затраты на обеспечение работы одного стационарного поста через $c_{i j}$.

Пусть также на исследуемом пространстве задана минимально допустимая вероятность обнаружения выбросов сетью стационарных постов $p_{\min }$.

Тогда многоэлементная задача размещения может быть сформулирована как задача выбора дополнительных постов и такого их расположения в точках выделенного пространства, при котором будут выполнены следующие условия:

1. Интегральная вероятность обнаружения загрязнения группой стационарных постов должна быть не менее установленного значения $p_{\text {min }}$ (обычно 0,95): $p_{\Sigma} \geq p_{\text {min }}$;

2. Расположение стационарных постов в местах наиболее вероятного обнаружения выброса с учетом розы ветров, характерной для данной местности, для множественных стационарных мест выброса должно быть таким, чтобы все источники загрязнения были представлены максимальным образом;

Интегральная эксплуатационная стоимость всех $N$ постов стационарного наблюдения

$$
S e=\sum_{m=1}^{N}\left(c_{i j}\right)_{m}
$$

не должна превышать суммарный ожидаемый ущерб $M s$ от рассматриваемых стационарных источников загрязнения: $\mathrm{Se} \leq \mathrm{Ms}$. Эксплуатационная стоимость стационарных постов наблюдения в течение времени нормирования определяется на основе технико-экономического анализа, а ожидаемый ущерб регламентирован стоимостью квоты выбросов и размером штрафных санкций их превышения. Так как величина превышения квоты на прогнозируемый период неизвестна, то допускается принимать ее равной размеру превышений за предыдущий отчетный период $[7,8]$. Сверхквотируемые выбросы от стационарных источников для прогнозируемого периода можно отнести к стохастической компоненте загрязнения. В качестве исходных данных для анализа существующей структуры сети мониторинга на уровне территории необходима следующая информация [3]:

- план территории;

- пространственное распределение промышленных предприятий (с указанием типа технологий, потенциально приводящих к значительным выбросам загрязняющих веществ, и существующих средств контроля выбросов);

- данные о параметрах и выбросах из всех значительных точечных источников загрязнения и основных рассредоточенных источников;
- пути прохождения и интенсивность основных транспортных потоков;

- топографические и метеорологические данные, с которыми могут быть связаны рассеивание выбросов и перенос загрязнения;

- пространственное распределение плотности населения, проживающего на данной территории;

- основные характеристики населения на различных территориях, включая состояние здоровья, распределение по возрасту, и т.д. (на последнее может указывать расположение больниц, школ, домов престарелых и т.д.).

\section{Результаты исследований. Методика} рациона^ьного расположения стационарных постов наб^юАения

Методика размещения стационарных постов $[4,5]$ состоит из 3 самостоятельно связанных друг с другом частей, направленных на последовательное сужение зоны поиска наилучших мест размещения, и базируется на использовании различных подходов, обеспечивающих:

1. определение зоны возможного превышения предельно допустимых концентраций примесей;

2. моделирование распределения концентраций загрязнений в приземном слое воздуха;

3. определение мест размещения постов на основании расчетов пространственной изменчивости загрязнений.

\section{Опрелеление зоны возможного превышения преАеАьно $\triangle$ опустимых коншентраший примесей}

Для мониторинга выбросов в атмосферу загрязняющих веществ от стационарных промышленных источников с известными характеристиками выбросов необходимо определить пространство, для которого возможно превышение предельно допустимых концентраций примесей (рис. 1).

Такую информацию можно получить путем предварительного моделирования выбросов с использованием известных моделей рассеяния примеси в атмосфере, адекватных рассматриваемым процессам выбросов, характерных для рассматриваемых стационарных техногенных источников эмиссии примеси [5-9]. К таким моделям можно отнести гауссовы модели рассеяния нейтральных и тяжелых газов.

Моделирование рассеяния от стационарных источников позволяет определить массив распределения максимальных концентраций $c_{m}(x, y, z)$ в пространстве в районе действия источника загрязнения. 


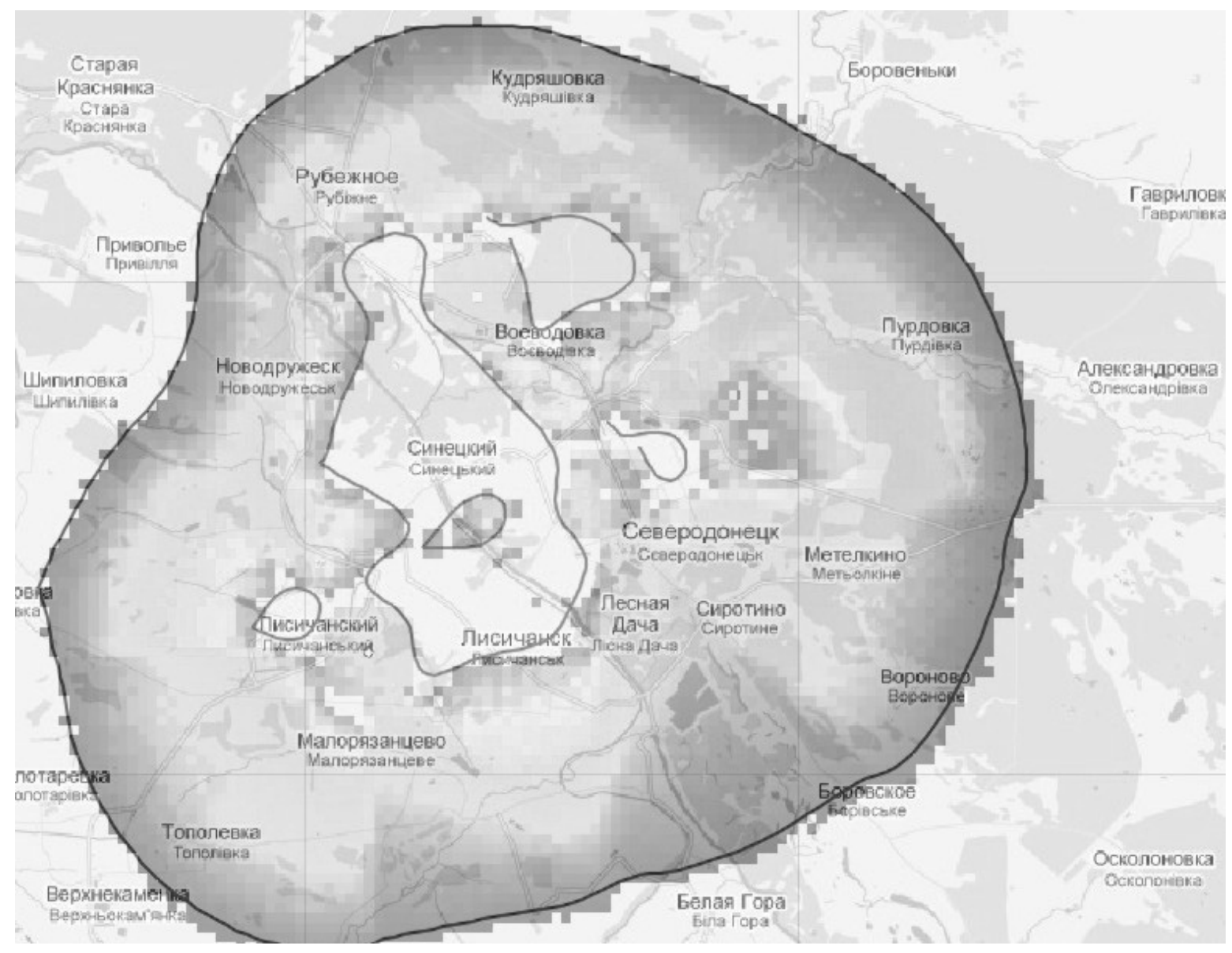

Рис. 1. Зона возможного превышения предельно допустимых концентраций примесей

Входные данные приведенных методик содержат $n$ независимых (совместных) стохастических показателей погодных условий и производительности источника (скорость ветра, класс стабильности атмосферы, направление ветра, мощность источника и др.). Сочетание количественных значений стохастических показателей определяют вероятность реализации распределения концентраций в пространстве (1):

$$
x \in\left[x_{\min }, x_{\max }\right] ; y \in\left[y_{\min }, y_{\max }\right] ; z \in\left[z_{\min }, z_{\max }\right] \text { (1) }
$$

где $x_{\min }, x_{\max }$ - максимальная и минимальная координата зоны определения концентраций по оси $x$;

$y_{\min }, y_{\max }-$ максимальная и минимальная координата зоны определения концентраций по оси у;

$z_{\min }, z_{\max }$ - максимальная и минимальная координата зоны определения концентраций по оси $z$;

В результате моделирования получаем массив $c_{m}(x$, $y, x)$ с учетом розы ветров данной местности. Тогда возможно выделить пространство, в котором концентрация превышает предельно допустимое значение. В пределах этого пространства необходимо проводить замеры.

\section{Определение пространственной изменчивости загрязнения}

Для получения информации об ожидаемых концентрациях загрязнителей используются известные модели распространения примеси в атмосфере от стационарных источников, с помощью которых вычисляется пространственно-временное распределение концентрации примеси при заданных условиях выброса для заданного источника загрязняющей примеси.

На рисунке 2 отображается моделируемое распределение максимальных концентраций загрязнителя при выбросе от одиночного стационарного источника загрязнения, превышающих ПДК (предельно допустимой концентрации для выбранного для мониторинга вещества опасной примеси).

Модель дисперсии примеси в атмосфере известна и представлена в работах [5, 10-13] и также программных кодах EFFEKT, ALOHA, RIZEX-2 и других. Входные данные для моделирования распределения концентраций в пространстве являются стохастическими $\left(b_{u}\right)$ : направ- 


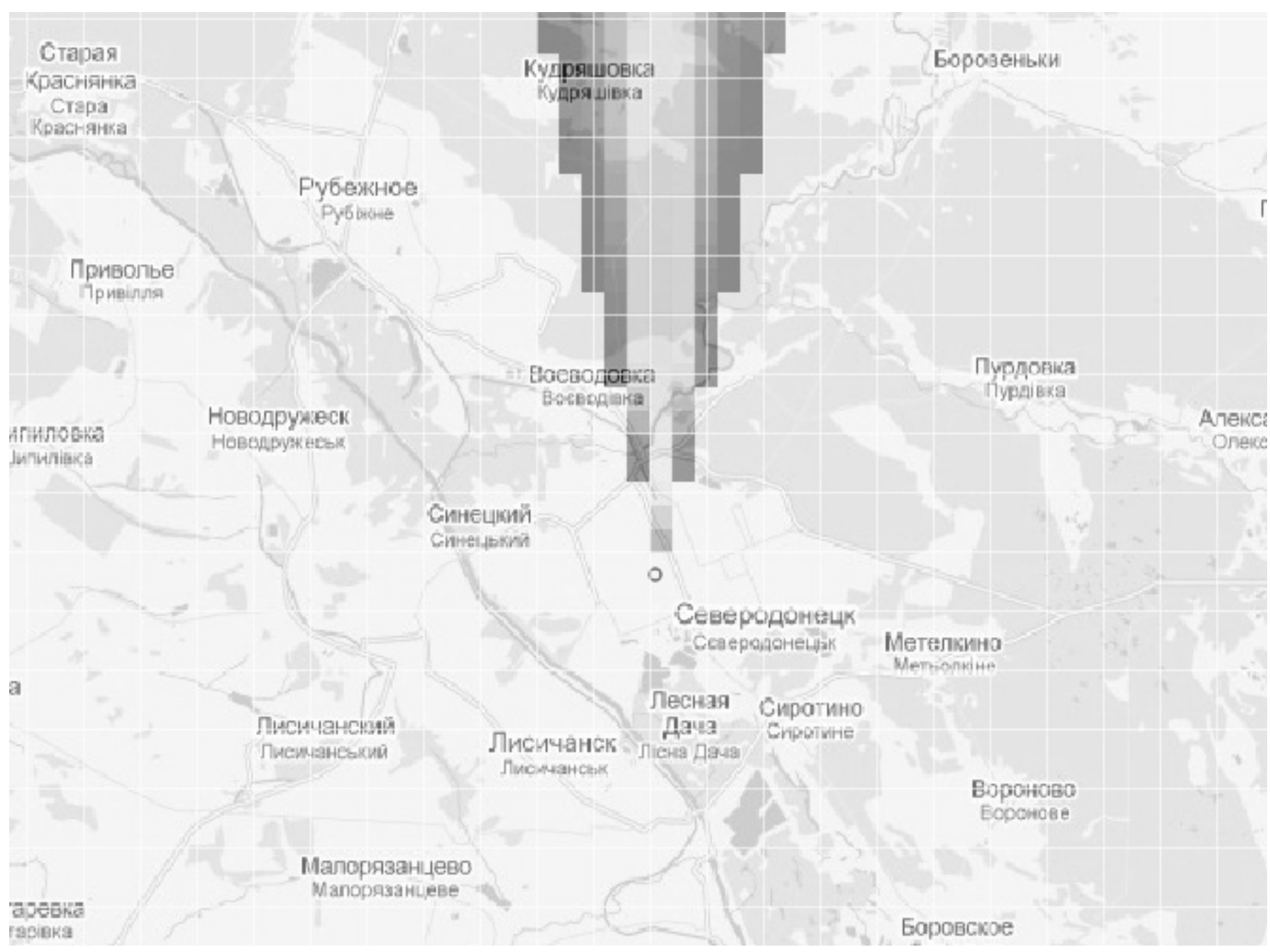

Рис. 2. Распределение максимальных концентраций (выше ПДК) от одного источника загрязнения при заданных погодных условиях

ление ветра; скорость ветра; стабильность атмосферы (инверсия, конвекция, изотермия); мощность источника загрязнения и др. Каждый стохастический $i$-й параметр характеризуется вероятностью нахождения в пределах некоторых диапазонов его изменения. Любой набор вероятных входных параметров исключает проявление другого набора, следовательно, объединяется по логической связи «исключающем ИЛИ». Это значит, что при наличии вектора фазовых переменных $\vec{\Phi}_{i}$ входных данных для каждого источника, их значения распределяются с различной вероятностью по диапазонам полного набора значений. Метеорологические данные, присущие данной местности характеризуются «розой ветров». Роза ветров, построенная по реальным данным наблюдений, позволяет по длине лучей построенного многоугольника выявить вероятность направлений ветра, а также вероятность скорости на высоте 10 метров. На рисунке 3 представлена диаграмма для 16 секторов направлений ветра. Существуют данные для 4, 8 и 16 сек- торов. Наиболее распространенные данные для 8 секторов, но предпочтительнее для 16.

Роза ветров строится на основании данных измерений, полученных в течение определенного периода и представленные в табличном виде на рисунке 4.

Данные для розы ветров, число секторов и классов ветра редактируется и задается в соответствии с доступными исследователю данными. Рекомендуется использовать фиксированное, представленное в таблице число диапазонов скоростей ветра. При скорости ветра более 7 м/с интенсивность рассеяния облака достаточно высока и угроза от него существенно уменьшается. Эти данные для розы ветров можно получить напрямую из метеорологических данных или провести замеры (собрать данные иных замеров) и обработать их для наполнения таблиц по результатам наблюдений следующим способом: 


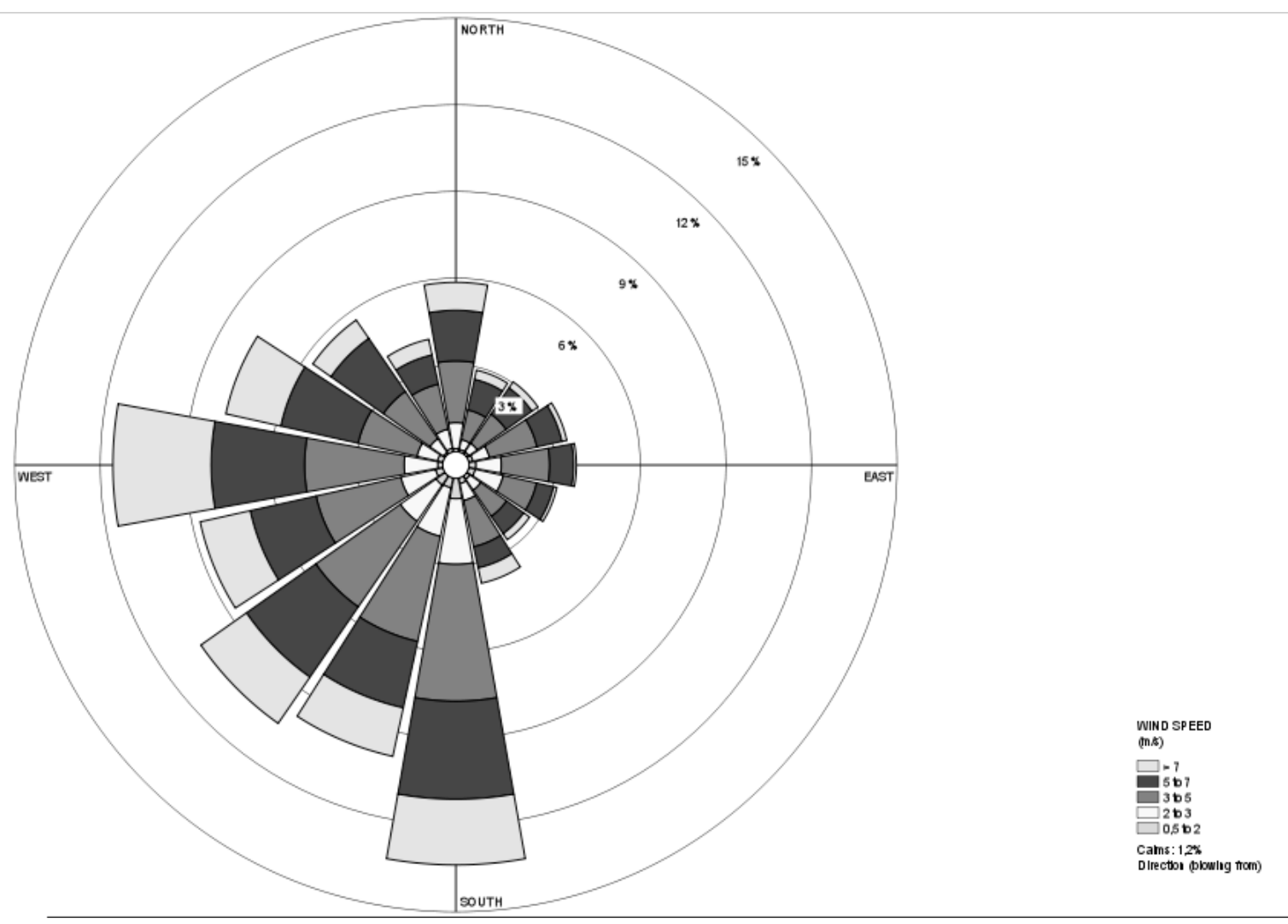

Рис. 3. Роза ветров для выбранной местности (г. Северодонецк)

\begin{tabular}{|c|c|c|c|c|c|c|c|}
\hline & $\begin{array}{c}\text { Directions / } \\
\text { Wind Classes }(\mathrm{m} / \mathrm{s})\end{array}$ & $0,5-2$ & $2-3$ & $3-5$ & $5-7$ & $>=7$ & Total \\
\hline 1 & $348,75-11,25$ & 9 & 78 & 185 & 152 & 84 & 508 \\
\hline 2 & $11,25-33,75$ & 8 & 30 & 91 & 94 & 28 & 251 \\
\hline 3 & $33,75-56,25$ & 8 & 31 & 107 & 96 & 22 & 264 \\
\hline 4 & $56,25-78,75$ & 13 & 51 & 148 & 78 & 15 & 305 \\
\hline 5 & $78,75-101,25$ & 21 & 77 & 147 & 73 & 8 & 326 \\
\hline 6 & $101,25-123,75$ & 20 & 87 & 104 & 53 & 10 & 274 \\
\hline 7 & $123,75-146,25$ & 12 & 40 & 96 & 63 & 22 & 233 \\
\hline 8 & $146,25-168,75$ & 17 & 52 & 142 & 65 & 47 & 323 \\
\hline 9 & $168,75-191,25$ & 61 & 197 & 411 & 294 & 197 & 1160 \\
\hline 10 & $191,25-213,75$ & 30 & 149 & 323 & 204 & 149 & 855 \\
\hline 11 & $213,75-236,25$ & 33 & 133 & 313 & 257 & 169 & 905 \\
\hline 12 & $236,25-258,75$ & 23 & 108 & 264 & 205 & 156 & 756 \\
\hline 13 & $258,75-281,25$ & 15 & 102 & 303 & 284 & 299 & 1003 \\
\hline 14 & $281,25-303,75$ & 17 & 64 & 183 & 241 & 171 & 676 \\
\hline 15 & $303,75-326,25$ & 6 & 50 & 173 & 194 & 67 & 490 \\
\hline \multirow[t]{4}{*}{16} & $326,25-348,75$ & 11 & 59 & 138 & 90 & 48 & 346 \\
\hline & Sub-Total & 304 & 1308 & 3128 & 2443 & 1492 & 8675 \\
\hline & Calms & & & & & & 109 \\
\hline & Total & & & & & & 8784 \\
\hline
\end{tabular}

Рис. 4. Данные розы ветров 
- фиксировать усредненные скорости и направления ветра в течение времени усреднения для каждого одиночного замера (время усреднения, например, принимать равным 1 часу). Чем большее число замеров будет произведено в течение времени определения розы ветров, тем более точный результат будет получен. Рекомендуется проводить ежедневные замеры в разное время суток в течение четырех - пяти лет;

сортировать полученный массив скоростей по возрастанию и вычислить массив, хранящий значения числа замеров, для которых значения скоростей ветра попадали в указанные диапазоны. Можно принять вероятность соответствующей скорости ветра как

$$
P_{v i}=\frac{n_{i}}{N}
$$

где $n_{i}$ - число замеров, попадающих в соответствующий $i$-й диапазон скоростей $(i \in 1 \ldots 5$ в соответствии с таблицей на рисунке 4), $N$ - общее число замеров;

провести аналогичную обработку результатов для направлений ветров, рассчитав вероятность того, что направление ветра попадет в соответствующий диапазон значений, получить массив вероятностей направлений ветра как

$$
P_{w d j}=\frac{k_{j}}{N^{\prime}}
$$

где $k_{j}$ - число замеров, попадающих в соответствующий $j$-й диапазон направлений $(j \in 1 \ldots 8$ в соответствии с таблицей на рисунке 4), $N$ - общее число замеров.

Алгоритм получения средних значений направления ветра для заданного времени усреднения замера по мгновенным значениям направлений таков: при наличии $\mathrm{n}$ значений направления ветра, полученных в течение времени усреднения $\mathrm{T}$, определяем среднее направление ветра $x$ (град) по минимальному отклонению среднего значения от всех мгновенных показателей $a_{i}$ (град), полученных в течение времени усреднения. Значения направления ветра получаем целочисленными величинами в градусах от направления на север.

Перебирая показатель $a_{i}$ для фиксированного $r_{i}$ получаем:

$$
\begin{aligned}
& \text { если }\left|x-a_{i}\right| \leq 180 \text {, то } r_{i}=\left|x-a_{i}\right|, \text { иначе } \\
& r_{i}=360-\left|x-a_{i}\right|
\end{aligned}
$$

рассчитываем сумму всех отклонений от величины $x$ :

$$
z n=\sum_{i=0}^{n} r_{i}
$$

проходим в цикле все значения $x$ от 0 до 359 градусов с шагом в 1 градус и вычисляем значение $\mathfrak{n}$ для каждого значения $x$;

в процессе цикла определяем минимальное значение $\mathfrak{n}$ для всех значений $x$ и соответствующее ему значение $x$.

Полученное значение является искомой величиной направления ветра, отклоняющейся от массива мгновенных значений на минимальную величину.

Данные могут быть получены и иными методами. В большинстве случаев данные метеорологических наблюдений доступны в виде стандартных файлов, представленных открытым форматом (например, LAKES FORMAT $[11,12])$. Итоговая условная вероятность реализации набора входных параметров определяется как (2):

$$
\mathrm{P}_{\mathrm{j}}=\prod_{\mathrm{k}=1}^{\mathrm{N}} \mathrm{P}_{\mathrm{k}},
$$

где $N$ - суммарное число независимых входных параметров, определяющих интенсивность распространения загрязнителя в пространстве.

Итоговая вероятность определения концентрации вещества, превышающей ПДК, выбранного для мониторинга от одного источника, в заданной точке поверхности земли (x, у) вычисляется как (3):

$$
\begin{aligned}
& P_{\text {пдк }}(x, y)=\sum_{\mathrm{j}=1}^{\mathrm{F}}\left(\prod_{k=1}^{N} P_{k}\right)_{j}, \\
& \text { где } F=\prod_{u} b_{u}-
\end{aligned}
$$

количество возможных сочетаний вероятных независимых входных параметров. Вероятность того, что ветер будет иметь направление от источника выброса в координату $(x, y)$ определяется из «розы ветров».

Например, для одиночного источника загрязнения итоговое поле условной вероятности обнаружения концентраций, превышающих ПДК (при условии реализации выбросов заданной производительности от данного источника), ограниченное нижней вероятностью порядка 0,005 (рис. 5).

Пунктирной линией ограничена территория, внутри которой вероятность обнаружения выбросов от данного источника превышает минимальную 0,005, жирной пунктирной линией отмечено пространство, вероятность обнаружения загрязнителя в котором превышает мини- 


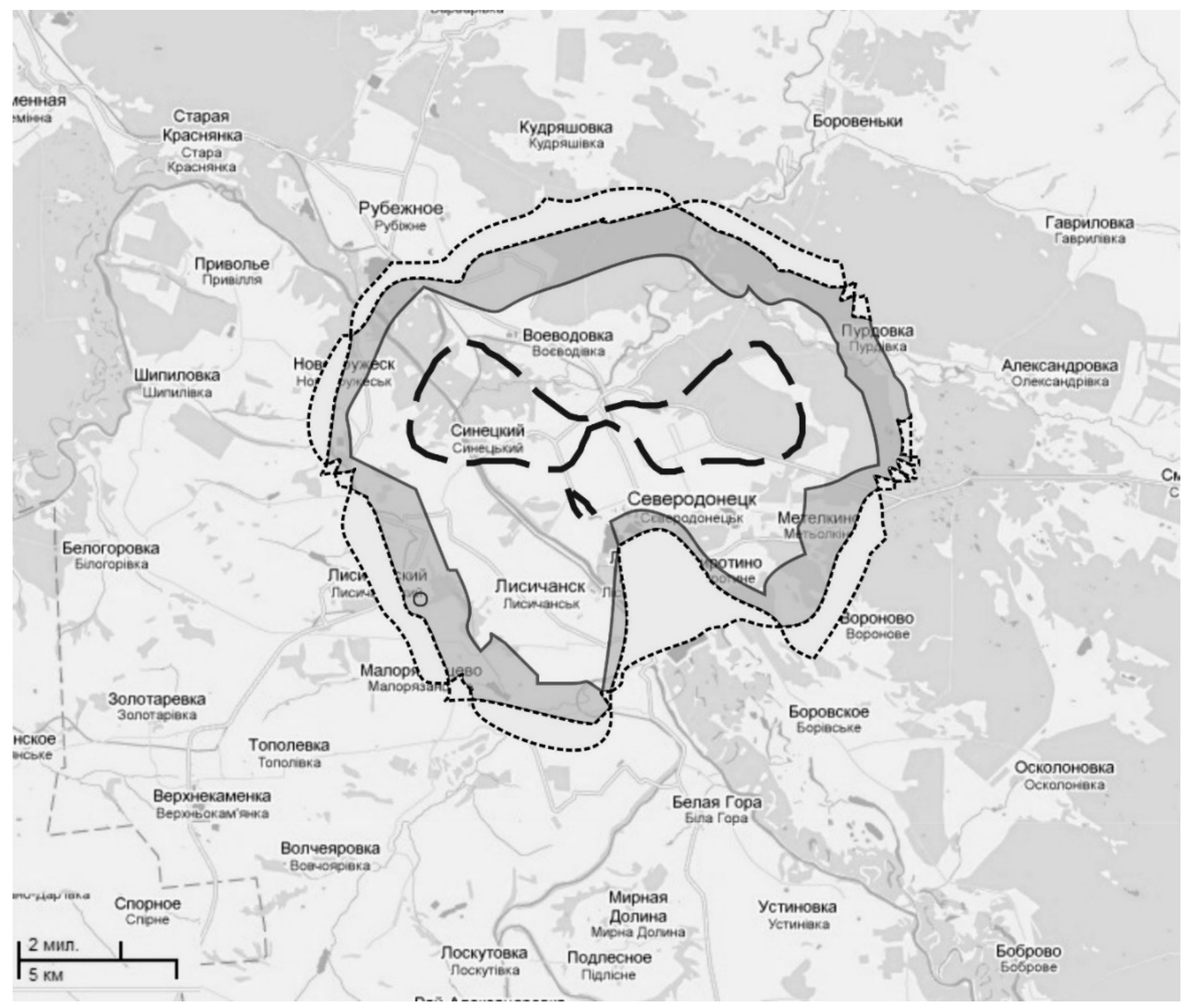

Рис. 5. Поле вероятности обнаружения концентраций, превышающих ПДК, от одного источника загрязнения (г. Северодонецк)

мально допустимую 0,05. Поле на рисунке 5 (и изолинии) получено для местности региона Северодонецк-Рубежное путем учета розы ветров и вероятностей выбросов примеси для данного источника опасности (ЧАО «Северодонецкое объединение «Азот»).

Сортировка вектора вероятностей (поля вероятности) по убыванию позволяет выделить набор координат, для которого итоговая вероятность $P_{\Sigma} \geq 0,95$. При этом необходим набор как минимум 4-х стационарных постов.

Если учитывать множественные стационарные источники загрязнения, необходимо построить такое же поле для всех учитываемых источников. Объединенное поле вероятности от множественных независимых источников NI интегрируется по формуле (4):

$$
P I_{\text {ПдК }}(x, y)=1-\prod_{1}^{N I}\left(1-\sum_{\mathrm{j}=1}^{\mathrm{F}}\left(\prod_{k=1}^{N} P_{k}(x, y)\right)_{j}\right)
$$

На рисунке 6 представлено такое поле для трех различных источников загрязнения.

Если число датчиков или стационарных постов известно, то при выборе узлов координатной сетки для их размещения, в которых вероятность обнаружения выброса максимальна, сумма этих вероятностей дает представление о надежности обнаружения выбросов. 


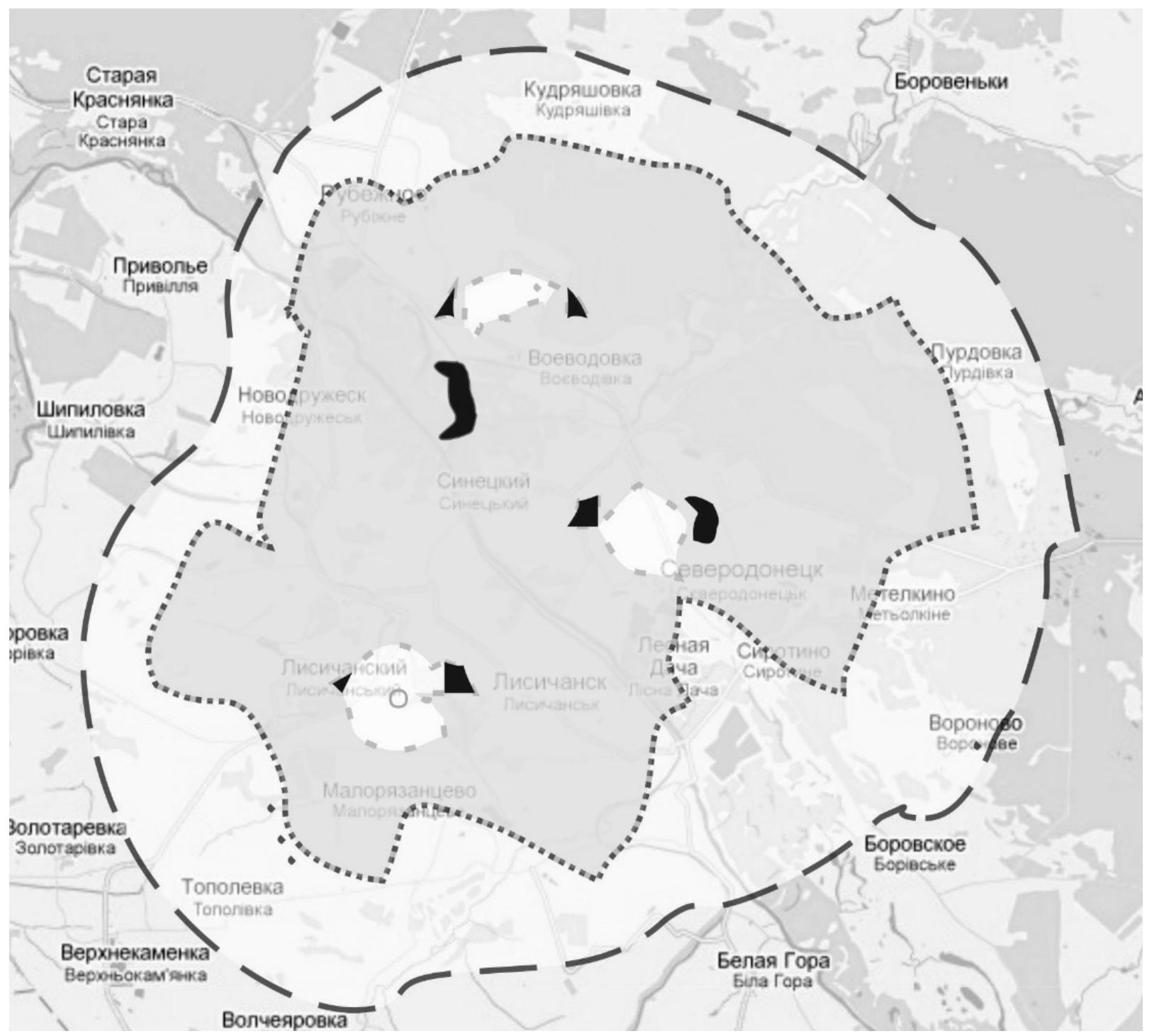

Рис. 6. Поле вероятности обнаружения концентраций, превышающих ПДК, от трех различных стационарных источников загрязнения

Если суммарная вероятность в этих узлах ниже приемлемой, величина которой определяется путем согласования с органами местного и государственного управления, то можно сделать вывод о необходимости дополнительных точек контроля за состоянием атмосферного воздуха. Суммируя значение вероятностей дальше по списку для координат дополнительных постов, вплоть до достижения приемлемого значения вероятности обнаружения выбросов дает нам необходимое число стационарных постов или датчиков.

\section{Зак^ючение}

1. Необходимым условием успешного проведения мероприятий по снижению концентрации вредных выбросов и работ по охране чистоты атмос- ферного воздуха является контроль, который дает объективную оценку работоспособности и эффективности всей деятельности по предупреждению загрязнения.

2. Сформулирована задача анализа структуры сети стационарных постов наблюдения атмосферного воздуха с целью повышения качества информированности о загрязнении при минимальных затратах.

3. Предложен метод размещения сети постов наблюдения, основанный на известных моделях рассеяния примеси в атмосфере, теории о регионализованной переменной и методах крайгинга для получения адекватных оценок о выбросах стационарных техногенных источников эмиссии примеси. 


\section{ЛИТЕРАТУРА}

1. Абрамов Ю.Ф. Региональная безопасность (эколого-информационный аспект) / Абрамов Ю.Ф., Душутин В.К., Сидоркина Е.В. - Иркутск: Изд-во Иркутского госуниверситета. 2003.- 200 c.

2. Анализ риска и его нормативное обеспечение / В.Ф. Мартынюк, М.В. Лисанов, Е.В. Кловач, В.И. Сидоров // Безопасность труда в промышленности.1995. - N11. - C. 55-62.

3. Белов П.Г. Системный анализ и моделирование опасных процессов в техносфере / Белов П.Г.— М.: Академия, 2003. — 512 с.

4. Богатиков В.Н. Построение дискретных моделей химико-технологических систем. Теория и практика. Апатиты / Богатиков В.Н., Палюх Б.В.- изд. Кольского научного центра, 1995. 164 с.

5. Варзар Р.Л. Разработка автоматизированной системы мониторинга загрязнения атмосферного воздуха: [Электронный ресурс] / Варзар Р.Л. — Режим доступа к работе: http://masters.donntu.edu.ua/2009/fvti/varzar/diss/index.htm.

6. Владимиров В.А. Оценка риска и управление техногенной безопасностью. Монография. / Владимиров В.А., Измалков В.И., Измалков А.В.— М.: ФИД «Деловой экспресс»-2002-184 с.

7. Гринин А.С. Экологический менеджмент: Учебное пособие для вузов / Гринин А.С., Орехов Н.А., Шмидхейни С. — М.: ЮНИТИ — ДАНА, 2001 — С. 6

8. Декларирование промышленной безопасности опасных производственных объектов: Сборник документов. Серия 27. Выпуск 3 / Колл. Авто.- 4-е изд.- М.: Федеральное государственное унитарное предприятие «Научно-технический центр по безопасности в промышленности», 2005.-296 с.

9. Израэль Ю.А. Экология и контроль состояния природной среды / Израэль Ю.А — М.: Гидрометеоиздат, 1984. — $560 \mathrm{C.}$

10. Кейн В.М. Оптимизация систем управления по минимаксному критерию / Кейн В.М. - М: Наука, 1985. — 248c.

11. Gessing R. Two-level hierarchical control for stochastic optimal resource allocation / Gessing R. //"Int. J. Contr."- 1985. — № 1.— P.161-175., Moor R.E. A survey of interval methods for differential equations //"Proc. — 23rd — IEEE Conf. Decis. and Contr., Las Vegas, Nev. — 1984. — Vol.3. — P. 1529-1535.

12. J.B. Fussel. Improving System Safety Through Risk Assessment / J.B. Fussell and others — Proceedings 1979 annual reliability and maintainability symposium, pp. 160-164

13. Ryazantsev A. The principles of information grouping in process diagnostic system / Ryazantsev A., Skarga-Bandurova I.— Радиоэлектронные и компьютерные системы. - 2006. — № 7(19).—C. 136-139.

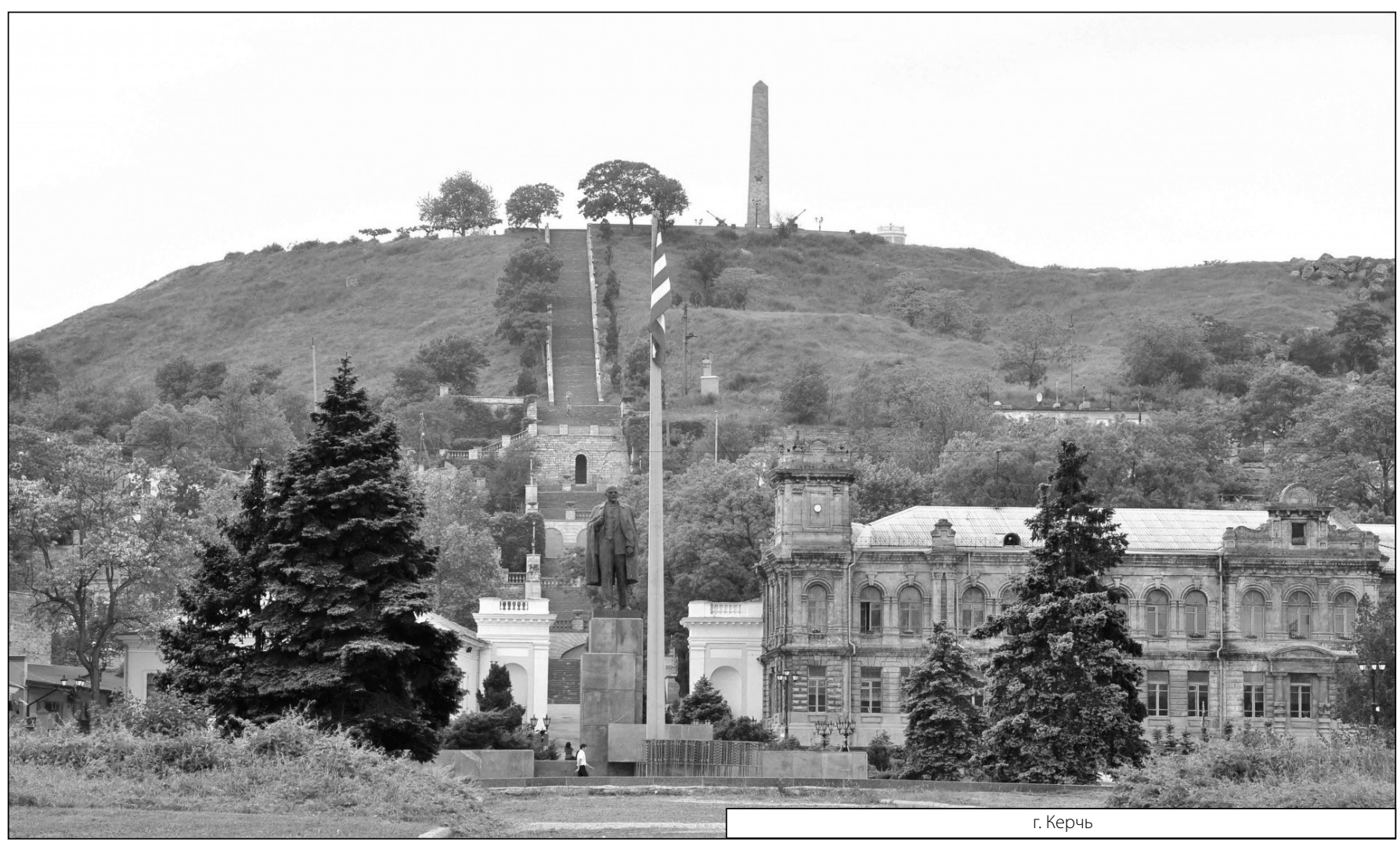

